September 93

\title{
Currents on Grassmann algebras
}

\author{
R. Coquereaux \\ Centre de Physique Théorique \\ CNRS Luminy - Case 907 \\ F 13288 Marseille Cedex 9 (France) \\ E. Ragoucy \\ Laboratoire de Physique Théorique ENSLAPP \\ B.P. 110 \\ F 74941 Annecy-le-Vieux Cedex (France)
}

\begin{abstract}
We define currents on a Grassmann algebra $\operatorname{Gr}(N)$ with $N$ generators as distributions on its exterior algebra (using the symmetric wedge product). We interpret the currents in terms of $\mathbb{Z}_{2}$-graded Hochschild cohomology and closed currents in terms of cyclic cocycles (they are particular multilinear forms on $G r(N)$ ). An explicit construction of the vector space of closed currents of degree $p$ on $\operatorname{Gr}(N)$ is given by using Berezin integration.
\end{abstract}

\section{Keywords :}

Grassmann algebras, super-algebras, supersymmetries, supermanifolds, Hochschild and cyclic cohomologies, De Rham currents.

September 1993

CPT - 93 / P.2935

ENSLAPP- 440/93 


\section{Introduction}

Roughly speaking, De Rham currents are distributions that can be pictured as possibly singular functions with several variables on a manifold. More precisely they extend the concept of distributions to the case of exterior forms. One can generalize currents to gradedcommutative calculus, i.e., in the simplest case, to Grassmann algebras. The analogy with the classical situation is useful but can be, at times, confusing, since the space of exterior forms over a Grassmann algebra in endowed with a symmetric wedge product in contradistinction with the usual exterior algebra; in particular the dimensionality of this space extends to infinity. With this warning in mind one notices that Grassmannian currents, like their classical counterpart in commutative geometry, i.e. the De Rham currents, and also like submanifolds may have boundary or not. The space of closed (i.e. without boundary) Grassmanian currents of degree $p$ over a Grassmann algebra with $N$ generators is a finite dimensional $\mathbb{Z}_{2}$-graded vector space.

In this paper, we show that these closed currents are in correspondence with $\mathbb{Z}_{2}$-graded cyclic cocycles over the Grassmann algebra: This is therefore a $\mathbb{Z}_{2}$-graded generalization of what is already known in the case of manifolds [1, 2]. The situation is actually quite simple because, in the case of Grassmann algebras, the super-commutative analogue of De Rham cohomology is trivial. In simple terms we shall determine the dimensionality of the space of closed currents and express them explicitly in terms of symmetric tensor (over the Grassmann algebra) whose Grassmann divergence is zero. The dimensionality of the space of cyclic cocycles (for general $p$ ) was already known after the work [5] and an explicit expression in the case $p=1$ was given for them was given in [3].

Our purpose here is

1) To interpret cyclic cocycles in term of closed Grassmannian currents

2) Build explicitly these objects in terms of symmetric tensors over the Grassmann algebra, using the Berezin integral.

\section{Differential forms over Grassmann algebras and Grass- mannian currents}

\subsection{Classical analogies}

From the algebraic point of view, it is convenient to consider the Grassmann algebra $G r(N)$ with $N$ generators $\theta^{\mu}, \mu \in\{1, \ldots, N\}$ as the graded-commutative analogue of the commutative algebra $A(N) \doteq C^{\infty}\left(\mathbb{R}^{N}\right)$ generated by the coordinates $x^{\mu}$. Clearly, $\mathbb{R}^{N}$ (or better, $A(N)$ at the algebraic level) is the simplest example of a manifold. In the same way, $G r(N)$ is the simplest example of a supermanifold.

In a course of elementary differential calculus, after having defined integration over $\mathbb{R}^{N}$, one builds the space $\Omega^{p} \doteq \Omega^{p}(A(N))$ of differential forms of degree $p$ and extend the definition of the integral to the whole of $\Omega \doteq \oplus_{p} \Omega^{p}$. The space of differential forms over $\mathbb{R}^{N}$ is built 
as the linear span (real coefficients) of products $d x^{\mu} \ldots d x^{\rho}$ with $d x^{\mu} d x^{\nu}=-d x^{\nu} d x^{\mu}$. Then one proves a basic tool of calculus, namely the Stokes' theorem that relates the integral of a differential $d \omega$ of the form $\omega$ over a particular subspace $X$ to the integral of the form $\omega$ over the boundary $\partial X$ of $X$. Finally, one proves the Poincaré Lemma: On $\mathbb{R}^{N}$, every closed (i.e. $d \omega=0) p$-form, $p>0$ is exact (i.e. $\exists \alpha$ with $\omega=d \alpha$ ). Thus, De Rham cohomology of $\mathbb{R}^{N}$ is trivial.

Physicists and mathematicians (also ingeneers!) know that, for many applications, one has to use distributions rather than functions. Usual distributions on $\mathbb{R}^{N}$ are therefore well known and are properly defined as linear forms over an appropriate class of test functions. One can generalize this concept of distributions to forms. Such distributions are usually called De Rham currents, distributional forms or simply "currents" [6] and are now a classical and necessary tool of analysis over $\mathbb{R}^{N}$ (or over manifolds). In simple terms, a current is a linear machine that produces a number out of a $p$-form. A simple example of a p-current $C$ can be obtained from any $p$-dimensional submanifold $S$ (more properly from any $p$-chain) by writing $\langle C, \omega\rangle \doteq \int_{S} \omega$. Completely antisymmetric contravariant tensors (so called " $p$-vectors") also define currents. Indeed, let $\mathcal{L} \doteq \operatorname{Der}(A(N))$ denote the space of derivations on $A(N)$; elements of $\mathcal{L}$ are vector fields $\chi=\chi^{\mu} \frac{\partial}{\partial x^{\mu}}$. Let us call $\mathcal{L}^{p}$ the space of $p$-vectors, with elements $\chi=$ $\chi^{\mu_{1} \mu_{2} \ldots \mu_{p}} \frac{\partial}{\partial x^{\mu_{1}}} \wedge \frac{\partial}{\partial x^{\mu_{2}}} \wedge \frac{\partial}{\partial x^{\mu_{p}}}$. As in the case of forms over $A(N)$, this wedge product of $p$-vectors is antisymmetric. Such a $p$-vector defines a $p$-current: Let $\omega=\omega_{\mu_{1} \mu_{2} \ldots \mu_{p}} d x^{\mu_{1}} \wedge d x^{\mu_{2}} \wedge d x^{\mu_{p}} \in \Omega^{p}$, one defines the current $C_{\chi}$ associated to $\chi$ via the equation

$$
\left\langle C_{\chi}, \omega\right\rangle \doteq \int_{\mathbb{R}^{N}} \chi^{\mu_{1} \mu_{2} \ldots \mu_{p}} \omega_{\mu_{1} \mu_{2} \ldots \mu_{p}} d^{N} x
$$

One can define a boundary operator $\partial$ mapping $p$-currents to $p-1$ as follows

$$
\langle\partial C, \omega\rangle=\langle C, d \omega\rangle
$$

Of course, currents can be closed (i.e. $\partial C=0$ ) or not. If we only consider "regular" objects, we can also associate $p$-vectors to currents and since we shall only stay at a purely formal level, i.e. forget about problems of analysis, this is what we shall do in most of the following and therefore identify $p$-currents and $p$-vectors. If $\chi$ denotes a $p$-vectors with corresponding current $C$, we shall call $\partial \chi$, with components $\frac{\partial}{\partial x^{\mu_{1}}} \chi^{\mu_{1} \mu_{2} \ldots \mu_{p}}$ the $(p-1)$-vector associated with the current $\partial C$. We shall often use the same notation $\chi$ or $C$ for both $p$-vectors and currents.

In classical differential calculus (commutative geometry), the next step is to generalize the above notions to the case of manifolds. As everybody knows, a new phenomenon appears: The Poincaré lemma may fail (a form that is closed is no longer necessarily exact!). However, our aim, in the present article, is to study (some) properties of Grassmann algebras related to currents and not to study the corresponding properties on arbitrary graded-commutative algebras. As stressed previously the former are simple graded-commutative analogues of $A(N)=C^{\infty}\left(\mathbb{R}^{N}\right)$ : They are the simplest "supermanifolds" and, exactly as for $\mathbb{R}^{N}$, De Rham cohomology is trivial. We can therefore stop here this paragraph devoted to classical analogies.

Let us stress the fact that the space $\Omega$ of exterior forms over $\mathbb{R}^{N}$ is isomorphic with $G r(N)$ is certainly true but can be confusing in the context of the present discussion. Let us repeat again that the analogy that we use here is between $A(N)$ and $\operatorname{Gr}(N)$. We now turn our attention to the Grassmanian analogue of $\Omega$, the algebra $\Lambda$ of exterior forms over $\operatorname{Gr}(N)$. 


\subsection{Grassmann algebras (a compendium of basic results and nota- tions)}

We summarize here some properties of Grassmann algebras and introduce a few notations that we shall need in the sequel. More properties can be found in [7] or [4].

A Grassmann algebra $\operatorname{Gr}(N)$ can be defined as a (real or complex) unital algebra with $N$ generators $\theta^{1}, \theta^{2} \ldots, \theta^{N}$ satisfying the relations $\theta^{\mu} \theta^{\nu}+\theta^{\nu} \theta^{\mu}=0$ and therefore $\left(\theta^{\mu}\right)^{2}=0$. In the following we choose the basic field as the field $\mathbb{C}$ of complex numbers. As a vector space, it has dimension $2^{N}$ and a possible basis is $\theta^{I}$ where $I$ is a multi-index $I \in$ $\{0, i, i j, \ldots, i j \ldots p, \ldots, 12 \ldots N\}$ with $1 \leq i<j<\ldots<p \leq N$ together with the convention $\theta^{I}=1$ when $I=0 . G r(N)={ }^{0} G r(N)+{ }^{1} G r(N)$ is a graded vector space but also a graded commutative algebra: Calling $\sigma_{a}$ the intrinsic grade of $a$ (i.e. 0 or 1 on even or odd product of $\theta^{\mu}$ resp.), we have $v u=(-1)^{\sigma_{u} \sigma_{v}} u v$. It is sometimes useful to decompose $\operatorname{Gr}(N)$ as $\operatorname{Gr}(N)=\mathbb{C}+\mathcal{N}$ where $\mathcal{N}$ denotes the space of nilpotents elements of $\operatorname{Gr}(N)$ and to notice that $\mathcal{N}$ is an ideal in $\operatorname{Gr}(N)$. There is a canonical homomorphism $\tau$ from $G r(N)$ to $\mathbb{C}$, i.e., a character, defined by $\tau(1)=1$ and $\left.\tau\right|_{\mathcal{N}}=0$. An element $u \in G r(N)$ is invertible iff $\tau(u) \neq 0$. In this case, calling $\frac{u}{\tau(u)}=1+x$ one gets $(1+x)^{-1}=1+\sum_{k=1}^{N}(-1)^{k} x^{k}$.

The generating system $\left\{\theta^{\mu}\right\}$ used to define $\operatorname{Gr}(N)$ is by no means unique; it is clear that one can make a change of generating system by using an invertible matrix with scalar coefficients but we should stress that one can also obtain new generating systems for the algebra $\operatorname{Gr}(N)$ by choosing coefficients in $G r(N)$ itself rather than in $\mathbb{C}$. More precisely, one calls a chart (or frame) on $\operatorname{Gr}(N)$ a generating system $\left(\eta^{\mu}\right)_{\mu \in\{1, \ldots, N\}}$ such that the $\eta^{\mu}$ are odd and such that $\eta^{1} \eta^{2} \ldots \eta^{N} \neq 0$, one proves that these two conditions imply $\eta^{\mu} \eta^{\nu}+\eta^{\nu} \eta^{\mu}=0$. Let then $\{\theta\}$ be a frame of $\operatorname{Gr}(N)$, the corresponding Berezin integral is the element of the dual $\operatorname{Gr}(N)^{*}$ given by

$$
\int a d_{\theta} \doteq \frac{\partial}{\partial \theta^{N}} \frac{\partial}{\partial \theta^{N-1}} \ldots \frac{\partial}{\partial \theta^{1}} a
$$

where $a \in G r(N)$. In other words this Berezin integral is equal to $a_{1,2, \ldots, N}$, the coefficient of $a$ on the "top element" $\theta^{1} \theta^{2} \ldots \theta^{N}$. We have to stress the fact that the left hand side of the previous equation is defined by the right hand side; in particular the symbol $d_{\theta}$ has nothing to do with a 1 -form $d \theta$ : see section 2.3. If $\{\eta\}$ is another chart of $\operatorname{Gr}(N)$-that we obtain from $\{\theta\}$ by an invertible matrix with coefficients in ${ }^{0} G r(N)$, the corresponding Berezin integral is defined in the same way but we have the relation

$$
\int d_{\eta} a=\int d_{\theta}\left|\frac{\partial \theta}{\partial \eta}\right| \times a
$$

where

$$
\left|\frac{\partial \theta}{\partial \eta}\right| \doteq \operatorname{Det}\left[\frac{\partial \theta^{\mu}}{\partial \eta^{\nu}}\right] \in G r(N)
$$

The fact that both Berezin integrals associated with charts $\theta$ and $\eta$ are related in this way illustrates another interesting property of Grassmann algebras, namely the fact that the dual $\operatorname{Gr}(N)^{*}$ of $\operatorname{Gr}(N)$ is simply generated as a right module over $\operatorname{Gr}(N)$, in other words, if $\phi \in G r(N)^{*}$ is such that $\phi\left(\theta^{1} \theta^{2} \ldots \theta^{N}\right) \neq 0$ then $\phi$ generates $\operatorname{Gr}(N)^{*}$, i.e. $\phi \operatorname{Gr}(N)=\operatorname{Gr}(N)^{*}$. 


\subsection{Derivations and differential forms over $\operatorname{Gr}(N)$}

We call $\mathcal{L} \doteq \operatorname{Der}(\operatorname{Gr}(N))$ the space of (graded) derivations on $\operatorname{Gr}(N)$, i.e. the analogue of the space of vector fields on $\mathbb{R}^{N}$. Calling $\theta=\left\{\theta^{1}, \ldots, \theta^{N}\right\}$ a chart in $\operatorname{Gr}(N)$ we notice that $\mathcal{L}$ is a left $\operatorname{Gr}(N)$-module (actually a free module) and that $\left\{\frac{\partial}{\partial \theta^{1}}, \ldots, \frac{\partial}{\partial \theta^{N}}\right\}$ is a basis of the module $\mathcal{L}$. In other word, an arbitrary element $\xi$ of $\mathcal{L}$ can be written $\xi=a^{\mu} \frac{\partial}{\partial \theta^{\mu}}$ with $a^{\mu} \in \operatorname{Gr}(N)$. As a vector space, $\mathcal{L}$ has a basis $\left\{\theta^{I} \frac{\partial}{\partial \theta^{\mu}}\right\}$ where $I$ is a multi-index (with $2^{N}$ possible values) and $\mu \in\{1,2 \ldots, N\}$ and it is also a graded vector space: $\mathcal{L}={ }^{0} \mathcal{L} \oplus^{1} \mathcal{L}$, with $\sigma_{\left(\theta^{I} \frac{\partial}{\left.\partial \theta^{\mu}\right)}\right.} \doteq \operatorname{card}(I)+1 \bmod 2$. Notice that, in particular, $\frac{\partial}{\partial \theta^{\mu}}$ is odd.

In the classical (commutative) case the space of vector fields is not only a $A(N)$-module but also a Lie algebra. Here the situation is analogous: $\mathcal{L}$ is a Lie superalgebra for the bracket $[\xi, \eta]=\xi \eta-(-1)^{\sigma_{\xi} \sigma_{\eta}} \eta \xi$ and, in particular $\frac{\partial}{\partial \theta^{\mu}} \frac{\partial}{\partial \theta^{\nu}}+\frac{\partial}{\partial \theta^{\mu}} \frac{\partial}{\partial \theta^{\mu}}=0$.

We call $\mathcal{L}^{*}$ the dual of $\mathcal{L}$ on $\operatorname{Gr}(N)$ and denote by $d \theta^{\mu}$ the elements of $\mathcal{L}^{*}$ defined by $<d \theta^{\mu}, \xi>=(-1)^{\sigma_{\xi}} \xi\left(\theta^{\mu}\right)$, in particular $<d \theta^{\mu}, \frac{\partial}{\partial \theta^{\nu}}>=-\delta_{\nu}^{\mu}$. An arbitrary element $\lambda$ of $\mathcal{L}^{*}$ is an arbitrary linear combination of the $\theta^{I} d \theta^{\mu}$. For an arbitrary element $a \in G r(N)$, one defines the $d$ operator as $d a=d \theta^{\mu} \frac{\partial}{\partial \theta^{\mu}} a \in \mathcal{L}^{*}$. Notice that $\mathcal{L}^{*}$ is also a left $G r(N)$-module. $\mathcal{L}^{*}$ is also a graded vector space and we define $\sigma_{\left(\theta^{I} d \theta^{\mu}\right)} \doteq \operatorname{card}(I) \bmod 2$. Notice that this definition of the grading makes the $d \theta$ 's even as we shall discuss it now.

We now call $\Lambda^{k} \doteq \Lambda^{k}(\mathcal{L}, G r(N))$ the space of $G r(N)$-valued, graded alternate $G r(N)$ linear $k$-forms over $\mathcal{L}$. Notice that $\Lambda^{1}=\mathcal{L}^{*}$. Elements of $\Lambda \doteq \sum_{0}^{\infty} \Lambda^{k}$ can be multiplied and $\Lambda$ becomes the Grassmann analogue of the usual algebra of exterior forms in $\mathbb{R}^{N}$. This space can also be considered as the exterior algebra over $\mathcal{L}^{*}$ but the wedge product between 1 -forms is now symmetric. Actually this is quite natural: 1-forms anti-commute in commutative geometry but they commute in anti-commutative geometry! In other words (and in practice) it is enough to remember that $d$ is odd, as well as the $\theta$ 's so that $d \theta$ is even and we can use the usual Milnor'sign rule. To avoid confusion, we denote this product ' $V$ ' in the sequel, and write for instance $d \theta^{\mu} \vee d \theta^{\nu}=d \theta^{\nu} \vee d \theta^{\mu}$ There is a only one $d$ operator of square 0 that extends the $d$ of the one-forms to the whole of $\Lambda$ and this space becomes then a $\mathbb{Z}_{2}$-graded and bigraded differential algebra (the bigrading is with respect to $\mathbb{N}$ ). Indeed, an arbitrary element of $\Lambda^{k}$ can be written $\lambda=\lambda_{\mu_{1} \mu_{2} \ldots \mu_{k}} d \theta^{\mu_{1}} \vee \ldots \vee d \theta^{\mu_{k}}$ where $\lambda_{\mu_{1} \mu_{2} \ldots \mu_{k}}$ is a covariant (down indices) symmetric tensor with coefficients in $\operatorname{Gr}(N)$, therefore $\lambda_{\mu_{1} \mu_{2} \ldots \mu_{k}}=\sum_{I} \lambda_{\mu_{1} \mu_{2} \ldots \mu_{k}}^{I} \theta^{I}$ where $\theta^{I}$ is a multi index. Each single term of this last sum is of the kind $\lambda_{\mu_{1} \mu_{2} \ldots \mu_{k}}^{i_{1} \ldots i_{p}} \theta^{i_{1}} \ldots \theta^{i_{p}}$ and this is why we have an $\mathbb{N}$-bigrading $(k, p)$. Because of the commutativity between the $d \theta$ themselves, graded commutativity of the wedge product depends only the $\mathbb{Z}_{2}$ grading $p \bmod 2$. One can also introduce an interior product and a Lie derivative in $\Lambda$ but we shall not use those concepts and we refer to [8] and [ [4] for more details. What is important for us is that $d=d \theta^{\mu} \frac{\partial}{\partial \theta^{\mu}}$; therefore, the above notation we can write $\omega \doteq d \lambda$ with

$$
\omega=\frac{1}{(k+1) !} \omega_{\mu_{0} \mu_{1} \ldots \mu_{k}} d \theta^{\mu_{0}} \vee \ldots \vee d \theta^{\mu_{k}}
$$

and

$$
\omega_{\mu_{0} \mu_{1} \ldots \mu_{k}} \doteq \partial_{\left[\mu_{0}\right.} \lambda_{\left.\mu_{1} \mu_{2} \ldots \mu_{k}\right]}
$$


where the symbols [...] denote complete symmetrization and where

$$
\partial_{\mu_{0}} \lambda_{\mu_{1} \mu_{2} \ldots \mu_{k}}=\sum_{i_{1}, \ldots, i_{p}} \sum_{s=i_{1}}^{i_{p}}(-1)^{(s+1)} \lambda_{\mu_{1} \ldots \mu_{k}}^{i_{1} \ldots i_{s} \ldots i_{p}} \delta_{\mu_{0}}^{i_{s}} \theta^{i_{1}} \ldots \hat{\theta}^{\hat{i}_{s}} \ldots \theta^{i_{p}}
$$

As usual, the hat on $\theta^{i_{s}}$ means that this factor has been omitted in the product. Notice that because of the symmetry properties of the $\vee$ product, the family $d \theta^{\mu_{1}} \vee \ldots \vee d \theta^{\mu_{k}}$ is a generating family for vectors, but is not a basis of $\Lambda^{k}$, unless we impose an ordering constraint like $\mu_{1} \leq \mu_{2} \leq \ldots \leq \mu_{k}$ (this is analogous to the situation prevailing in the case of usual exterior forms).

At the dual level, currents - that we take as $p$-vectors - will also be represented by contravariant (up-indices) symmetric tensors with coefficients in $G r(N)$, i.e. $\phi=\phi^{\mu_{1} \ldots \mu_{k}} \partial_{\mu_{1}} \vee$ $\ldots \vee \partial_{\mu_{k}}$. The pairing between $p$-vectors and $p$-forms is given by

$$
\langle\phi, \lambda\rangle \doteq(-)^{k} \int d_{\theta}^{N} \phi^{\mu_{1} \ldots \mu_{k}} \lambda_{\mu_{1} \ldots \mu_{k}}
$$

A well known result of elementary tensorial calculus (or of quantum mechanics of systems with identical bosonic particles) tell us that the space of symmetric tensors of order $p$ built on a vector space of dimension $N$ has dimension $C_{N+p-1}^{p}$, but in the present case, the coefficients belong to the Grassmann algebra $\operatorname{Gr}(N)$, itself of dimension $2^{N}$, therefore the space of differential forms over $\operatorname{Gr}(N)$ has dimension $\operatorname{dim}\left(\Lambda^{p}(\mathcal{L}, G r(N))=2^{N} C_{N+p-1}^{p}\right.$. Notice that, in contrast with the case of usual exterior forms over $\mathbb{R}^{N}$ or over a finite dimensional manifold, the space of all such forms (direct sum of all spaces $\Lambda^{p}$ for all values of $p$ ) is infinite dimensional since $\operatorname{dim}\left(\Lambda^{p}\right)$ grows with $p$.

The pairing between contravariant and covariant symmetric $\operatorname{Gr}(N)$-valued tensors (i.e. currents and forms) enables one to define a boundary operator $\partial$ already mentioned before. Writing $\langle\partial \psi, \lambda\rangle \doteq\langle\psi, d \lambda\rangle$ for a $p$-vector $\psi$ and a $(p-1)$-form $\lambda$ leads to

$$
\partial \psi^{\mu_{1} \ldots \mu_{p-1}}=\sum_{j=1}^{N} \partial_{j} \tilde{\psi}^{j \mu_{1} \ldots \mu_{p-1}}
$$

where " " denotes the involution of $G r(N)$ associated with the $\mathbb{Z}_{2}$ grading, i.e. a change of sign in front of odd monomials (for instance if $a=2 \theta^{3}+5 \theta^{1} \theta^{2}-\theta^{1} \theta^{2} \theta^{3}$ then $\tilde{a}=-2 \theta^{3}+$ $\left.5 \theta^{1} \theta^{2}+\theta^{1} \theta^{2} \theta^{3}\right)$.

Notice that a $\operatorname{Gr}(N)$-valued contravariant tensor $\phi^{\mu_{1} \mu_{2} \ldots \mu_{p}}$ defines a $\operatorname{Gr}(N)$-valued differential operator of degree $p$, namely $P_{\phi} \doteq \phi^{\mu_{1} \mu_{2} \ldots \mu_{p}} \partial_{\mu_{1}} \partial_{\mu_{2}} \ldots \partial_{\mu_{p}}$ where the product of symbols is now just the composition law $\partial_{\mu_{1}} \partial_{\mu_{2}} \ldots \partial_{\mu_{p}}$ but as $\partial_{\mu} \partial_{\nu}=-\partial_{\nu} \partial_{\mu}, P_{\phi}$ is identically zero when $\phi$ is symmetric. In what follow, we will use $p$-vectors $\phi=\phi^{\mu_{1} \ldots \mu_{p}} \partial_{\mu_{1}} \vee \ldots \vee \partial_{\mu_{p}}$ with a symmetric wedge product $\vee$. Differential operators and $p$-vector coincide only when $p=1$. In that case, the quantity $\partial \phi$, which is the boundary of $\phi$, can also be called the (graded) divergence of the operator $P_{\phi}$ with, of course, $\partial \phi=\operatorname{Div}\left(P_{\phi}\right)$. This identification is used, for instance in [3].

The last thing to study in this paragraph is the Grassmann analogue of De Rham cohomology of differential forms (or Grassmann homology of currents). Let us denote by 
$Z_{D R}^{p} \doteq\left\{\omega \in \Lambda^{p}, d \omega=0\right\}, B_{D R}^{p} \doteq\left\{\omega \in \Lambda^{p}, \omega=d \psi\right\}$ and $H_{D R}^{p} \doteq Z_{D R}^{p} / B_{D R}^{p}$. One can prove that this cohomology is trivial: $H_{D R}=0$. Indeed one can construct (see for instance [4) an operator $h$ from $\Lambda^{p}$ to $\Lambda^{p-1}$ such that $d h+h d=1$, therefore, given a closed form $\omega$, one defines $\psi=h \omega$ and shows that $\omega$ is exact $(\omega=d \psi)$ by using the previous relation between $d$ and $h$. From this point of view, Grassmann algebras are topologically trivial (as their "commutative" counterpart): The Poincaré lemma holds.

Before ending this paragraph, let us remark that the symbol $d_{\theta}$ appearing in the Berezin integral should not be considered as a shorthand for $d \theta^{1} \vee d \theta^{2} \ldots \vee d \theta^{N}$, at least not with the symmetric product $\vee$, as defined above, in mind. Indeed the law of change of variables in the Berezin integral shows that this integral should be transformed by a global -1 multiplicative constant upon a parity-odd change of chart such that

$$
\left\{\theta^{1}, \theta^{2}, \theta^{3}, \ldots, \theta^{N}\right\} \longrightarrow\left\{\theta^{2}, \theta^{1}, \theta^{3}, \ldots, \theta^{N}\right\}
$$

and this is clearly incompatible with a symmetric wedge product. One possibility is to introduce a new (antisymmetric) product - that one would call "." rather than " $\mathrm{V}$ " - between the $d \theta$ 's, just for the sake of making the notation $\int f(\theta) d \theta$ compatible with the law of change of variables. Another possibility is to refrain from writing a Grassmanian volume element in Berezin integrals, but this notation is now quite popular ... Let us only remember that the "natural" product between $d \theta$ 's is the symmetric one and that it is that one that we have been using and shall use in the following, even if we do not write explicitly the wedge symbol. Actually, Berezin himself, in his book [7] does not use the symbols $d \theta^{i}$ in his integrals but the symbol $d_{\theta^{i}}$ and warns the reader (footnote page 76 ) that he should not confuse the $d_{\theta^{i}}$ with the differential $d \theta^{i}$ : it is his notation that we have choosen, and we will write for the volume element:

$$
d_{\theta}^{N}=d_{\theta^{1}} d_{\theta^{2}} \ldots d_{\theta^{N}}
$$

\section{Hochschild and cyclic cohomologies for Grassmann algebras}

\subsection{Hochschild cohomology}

The definition of Hochschild cohomology of an arbitrary non-commutative algebra $A$ with values in the bimodule $A^{*}$-its dual- can be found, for instance in [四. The Hochschild $p$ cochains can be taken, in the present case, as arbitrary complex-valued $(p+1)$-linear forms over $\operatorname{Gr}(N)$. Their space will be denoted by $\mathcal{C}^{p}$. Notice that this space is naturally $\mathbb{Z}_{2^{-} \text {-graded }}$ $\left(\mathcal{C}^{p}={ }^{0} \mathcal{C}^{p} \oplus{ }^{1} \mathcal{C}^{p}\right)$ and that $\operatorname{dim} \mathcal{C}^{p}=\left(2^{N}\right)^{p+1}=2^{N(p+1)}$ with $\operatorname{dim}{ }^{0} \mathcal{C}^{p}=\operatorname{dim}{ }^{1} \mathcal{C}^{p}=2^{N(p+1)-1}$. The generalization of Hochschild cohomology to $\mathbb{Z}_{2^{2}}$ graded algebras can be found, for instance in [9] and the corresponding coboundary operator $b$ mapping $\mathcal{C}^{p}$ to $\mathcal{C}^{p+1}$ and satisfying $b^{2}=0$ is defined as follows:

$$
\begin{aligned}
{[b \Phi]\left(a_{0}, a_{1}, \ldots, a_{p}, a_{p+1}\right) \doteq } & \sum_{j=0}^{p}(-1)^{j} \Phi\left(a_{0}, \ldots, a_{j} a_{j+1}, \ldots, a_{p+1}\right) \\
& +(-1)^{p+1}(-1)^{\epsilon_{p+1}} \Phi\left(a_{p+1} a_{0}, a_{1}, \ldots, a_{p}\right)
\end{aligned}
$$


with

$$
\epsilon_{p+1}=\left(\sigma_{a_{p+1}}\right) \times \sum_{i=0}^{p} \sigma_{a_{i}}
$$

The space of Hochschild cocycles and coboundaries will be denoted by $Z^{p}$ and $B^{p}$ with cohomology $H^{p} \doteq Z^{p} / B^{p}$.

We want to show that, in the case of Grassmann algebras, Hochschild cocycles are in one to one correspondence with $p$-currents. We choose therefore

$$
\phi=\phi^{\mu_{1} \ldots \mu_{p}} \frac{\partial}{\partial \theta^{\mu_{1}}} \vee \frac{\partial}{\partial \theta^{\mu_{2}}} \vee \ldots \vee \frac{\partial}{\partial \theta^{\mu_{p}}}
$$

where $\phi^{\mu_{1} \ldots \mu_{p}} \in G r(N)$ is a symmetric tensor (remember that $\vee$ is symmetric). The corresponding Hochschild cochain $\Phi$ is defined by

$$
\begin{aligned}
\Phi\left(a_{0}, a_{1}, \ldots, a_{p}\right) & =\left\langle\phi, a_{0} d a_{1} \ldots d a_{p}\right\rangle \\
& =(-)^{\epsilon} \int d_{\theta}^{N} \phi^{\mu_{1} \ldots \mu_{p}} \\
\text { with } \epsilon & a_{0}\left(\partial_{\mu_{1}} a_{1}\right)\left(\partial_{\mu_{2}} a_{2}\right) \ldots\left(\partial_{\mu_{p}} a_{p}\right) \\
& =\frac{p(p+1)}{2}+\sum_{i=0}^{p}(p-i) \sigma_{a_{i}}
\end{aligned}
$$

It is a simple exercise to show that $b \Phi=0$, hence $\Phi$ is a Hochschild cocycle: $\Phi \in Z^{p}$.

Conversely, let $\Phi$ an arbitrary $p$-Hochschild cocycle. It has, a priori, no reason to be symmetric. We have to symmetrize it and define a $p$ current as:

$$
\left\langle\phi, a_{0} d a_{1} \ldots d a_{p}\right\rangle \doteq \sum_{s \in \mathcal{S}} \Phi\left(a_{0}, a_{s(1)}, \ldots, a_{s(p)}\right)
$$

Starting with some Hochschild cochain $\Psi$, one can show that if $\Phi=b \Psi$, then the current $\phi$ corresponding to this Hochschild coboundary $\Phi$ is zero. Indeed, using the definition of $b$ and, after the symmetrization process described in eq. (3.5), all the terms vanish pairwise. This implies that we can identify the space of Grassmannian currents (elements of $\left.\left(\Lambda^{p}\right)^{*}\right)$ with Hochschild cohomology $H^{p}$. As a consequence, $\operatorname{dim}\left(H^{p}\right)=2^{N} C_{N+p-1}^{p}$. Notice that identification of De Rham currents with Hochschild cocycles seems to be a quite general property, since it also holds in the classical case of manifolds [1] (with the difference, of course, that the dimensionality of $H^{p}$ grows with $p$ in the Grassmanian case whereas it is zero as soon as $p$ exceeds the dimension of the manifold in the classical-commutative-case). We do not study here the structure of general supermanifolds - Grassmann algebra being the simplest example- but we conjecture that, modulo an appropriate definition of currents generalizing the one given previously, the same result should hold.

Notice that a Grassmann algebra is both $\mathbb{Z}$-graded and $\mathbb{Z}_{2}$-graded; our previous construction of the correspondance between currents and Hochschild cocycles uses implicitely the underlying $\mathbb{Z}$-grading. Indeed, it uses the Berezin integral, but the value of this integral will change if we make a change of chart with coefficients in ${ }^{0} G r(N)$ rather than in $\mathbb{C}$. Such a change a chart would preserve the $\mathbb{Z}_{2}$-grading but modify the $\mathbb{Z}$-grading.

Finally, let us remark that it may help the reader to think of $\operatorname{Gr}(N)$ as the fermionic Fock space built over a fermionic system with $N$ levels (i.e. a complex vector space of dimension $N$ ) 
and of Hochschild $p$-cocycles as bosonic transitions associating a number to $(p+1)$ elements of the fermionic Fock space (transitions are of bosonic type because of the symmetry properties of the cocycles).

\subsection{Cyclic cohomology (results)}

The cyclicity operator $\lambda$, in the $\mathbb{Z}_{2}$-graded case, is defined by

$$
[\lambda \Phi]\left(a_{0}, a_{1}, \ldots, a_{p}\right) \doteq(-1)^{p}(-1)^{\epsilon_{p}} \Phi\left(a_{p}, a_{0}, \ldots, a_{p-1}\right)
$$

where

$$
\epsilon_{p} \doteq\left(\sigma_{a_{p}}\right) \times \sum_{i=0}^{p-1} \sigma_{a_{i}}
$$

and $\Phi$ is a cyclic cochain iff $\Phi o \lambda=\Phi$. The cyclic complex [1] is a subcomplex of Hochschild complex. In other words, cyclic cochains of degree $p$ on the Grassmann algebra are represented by $(p+1)$-linear forms on $\operatorname{Gr}(N)$-i.e. Hochschild cochains - which are cyclic; their space will be denoted $\mathcal{C}_{\lambda}^{p}$. A cyclic cochain is therefore characterized by the value that it takes on $(p+1)$ basis elements chosen among the $2^{N}$ that span $\operatorname{Gr}(N)$. Cyclic invariance shows therefore that $\operatorname{dim} \mathcal{C}_{\lambda}^{p}=2^{N(p+1)} /(p+1)$. Again this vector space is graded $\mathcal{C}_{\lambda}^{p}={ }^{0} \mathcal{C}_{\lambda}^{p} \oplus{ }^{1} \mathcal{C}_{\lambda}^{p}$ and $\operatorname{dim}{ }^{0} \mathcal{C}_{\lambda}^{p}=\operatorname{dim}{ }^{1} \mathcal{C}_{\lambda}^{p}=2^{N(p+1)-1} /(p+1)$. The space of cyclic cocycles and coboundaries will be denoted by $Z_{\lambda}^{p}$ and $B_{\lambda}^{p}$ with cohomology $H_{\lambda}^{p} \doteq Z_{\lambda}^{p} / B_{\lambda}^{p}$. In the case of manifolds, it can be shown [1] that $H_{\lambda}^{k}=\operatorname{Ker} \partial_{k} \oplus H_{D R}^{k-2} \oplus H_{D R}^{k-4} \oplus \ldots$, where $H_{D R}^{k}$ denotes the homology of De Rham currents (or of differential forms, at the dual level). When De Rham (co)homology is trivial one just gets $H_{\lambda}^{p}=\operatorname{Ker} \partial_{p}$ where $\partial_{p}$ is the boundary operator acting on currents. This result suggests, by analogy with the classical case, that one should find something similar in the Grassmanian case, i.e. we expect $H_{\lambda}^{p}$ to be essentially given by $K e r \partial_{p}$, where $\partial_{p}$ is the boundary operator acting on Grassmanian currents, as defined in section 3. By "essentially", we mean precisely the following:

$$
H_{\lambda}^{p}(G r(N))=\operatorname{Ker} \partial_{p} \oplus H_{\lambda}^{p}(\mathbb{C})
$$

where $H_{\lambda}^{p}(\mathbb{C})$ is equal to $\mathbb{C}$ if $p$ is even and 0 if $p$ is odd. This is what we show in the following. More precisely, we obtain the following results:

- Closed currents can be expressed as symmetric multilinear forms on $\operatorname{Gr}(N)$ which are of vanishing graded divergence (i.e. $\partial \psi=0$ ).

- By direct counting, the space of such currents of degree $p$ is a graded vector space

$$
\operatorname{Ker} \partial_{p} \doteq V^{p}(N)={ }^{0} V^{p}(N) \oplus{ }^{1} V^{p}(N)
$$

of dimensions $\operatorname{dim}{ }^{0} V^{p}(N)=2^{N-1} \gamma_{p}^{N}-(-1)^{p}$ and $\operatorname{dim}{ }^{1} V^{p}(N)=2^{N-1} \gamma_{p}^{N}$ with

$$
\begin{aligned}
\gamma_{p}^{N} & =\sum_{i=1}^{p+1}(-1)^{(i-1)} C_{N+p-i}^{p-(i-1)} \\
& =C_{N+p-1}^{p}-C_{N+p-2}^{p-1}+C_{N+p-3}^{p-2}-\ldots+(-)^{p} C_{N-1}^{0}
\end{aligned}
$$


- To any closed current, one can associate a cyclic cocycle.

- When $p$ is even, there will be a (unique, up to scalar multiple) canonical cyclic cocycle of degree $p$ that we shall call $\tau$. This cocycle cannot be expressed in terms of closed currents.

- To any cyclic cocycle which has no component along $\tau$ one can associate a closed current.

An explicit basis of $H_{\lambda}^{p}$ can therefore be found by using the previous results along with Berezin integration. The cyclic cohomology groups are therefore $H_{\lambda}^{p}(G r(N))=H_{\lambda}^{p}(\mathbb{C}) \oplus V^{p}(N)$ where $H_{\lambda}^{p}(\mathbb{C})$ denotes the cyclic cohomology of complex numbers (it is known that $H_{\lambda}^{2 s}(\mathbb{C})=\mathbb{C}$ and $H_{\lambda}^{2 s+1}(\mathbb{C})=0$ ) and where $V^{p}(N)$ is the graded vector space introduced previously. The dimensionality of $H_{\lambda}^{p}(\operatorname{Gr}(N))$ has been calculated in the literature by using Künneth-like formulae [5]. The novel feature here is the relation of cyclic cocycles to Grassmanian currents and their explicit expression in terms of $G r(N)$-valued symmetric multilinear forms with vanishing graded divergence; as a by-product, we recover the known result concerning the dimension of $H_{\lambda}^{p}(G r(N))$.

Notice that the dimensionality of the space of non-trivial cyclic cocycles is given (up to a global factor $2^{N}$ and up to 1 when $p$ is even) by the alternated partial sums along NW-SE diagonals of the Pascal triangle.

\subsection{Closed currents, divergence-free Grassmann- valued contravari- ant symmetric tensors and cyclic cocycles}

We already showed that a closed current of degree $p$ (or a closed $p$-vectors) can be written in terms of a divergence-free differential operator of degree $p$, i.e. in terms of a Grassmann valued contravariant symmetric tensor $\phi^{\mu_{1} \mu_{2} \ldots \mu_{p}}$ satisfying $\sum_{j=1}^{N} \partial_{j} \tilde{\phi}^{j \mu_{1} \ldots \mu_{p}}=0$. It remains to show that it defines a cyclic cocycle $\Phi$. We already know (because it is a current) that it defines

a Hochschild cocycle. The only thing to check is that it is cyclic. The following sequence of equalities establishes the claimed property.

$$
\begin{aligned}
\Phi\left(a_{0}, a_{1}, \ldots, a_{p}\right) & =\int d_{\theta}^{N} \phi^{\mu_{1} \ldots \mu_{p-1} \mu_{p}} a_{0}\left(\partial_{\mu_{1}} a_{1}\right) \ldots\left(\partial_{\mu_{p-1}} a_{p-1}\right)\left(\partial_{\mu_{p}} a_{p}\right) \\
& =(-1)^{p}(-1)^{\epsilon_{p}} \int d_{\theta}^{N} \phi^{\mu_{1} \ldots \mu_{p-1} \mu_{p}}\left(\partial_{\mu_{p}} a_{p}\right) a_{0}\left(\partial_{\mu_{1}} a_{1}\right) \ldots\left(\partial_{\mu_{p-1}} a_{p-1}\right) \\
& =+(-1)^{p}(-1)^{\epsilon_{p}} \int d_{\theta}^{N} \phi^{\mu_{1} \mu_{2} \ldots \mu_{p-1} \mu_{p}} a_{p}\left(\partial_{\mu_{p}} a_{0}\right)\left(\partial_{\mu_{1}} a_{1}\right) \ldots\left(\partial_{\mu_{p-1}} a_{p-1}\right) \\
& =(-1)^{p}(-1)^{\epsilon_{p}} \int d_{\theta}^{N} \phi^{\mu_{2} \mu_{3} \ldots \mu_{p} \mu_{1}} a_{p}\left(\partial_{\mu_{1}} a_{0}\right)\left(\partial_{\mu_{2}} a_{1}\right) \ldots\left(\partial_{\mu_{p}} a_{p-1}\right) \\
& =(-1)^{p}(-1)^{\epsilon_{p}} \int d_{\theta}^{N} \phi^{\mu_{1} \mu_{2} \ldots \mu_{p-1} \mu_{p}} a_{p}\left(\partial_{\mu_{1}} a_{0}\right)\left(\partial_{\mu_{2}} a_{1}\right) \ldots\left(\partial_{\mu_{p}} a_{p-1}\right) \\
& =[\lambda \Phi]\left(a_{0}, a_{1}, \ldots, a_{p}\right]
\end{aligned}
$$

Where the symbol $\epsilon_{p}$ has the same meaning as before. In the above calculation, we use, in turn the following properties 
1. The fact that a current $\phi$ defines a Hochschild cocycle $\Phi$.

2. (Graded) Commutation of the element $\partial_{\mu_{p}} a_{p}$ with the other elements.

3. Integration by part (with a " + " sign) and the fact that $\phi$ is closed, i.e. $\partial \phi=0$.

4. Relabelling of indices.

5. The fact that $\phi^{\mu_{1} \ldots \mu_{p}}$ is symmetric.

6. Definition of the cyclicity operator $\lambda$.

Notice that the Hochschild property $b \Phi=0$ is translated in the fact that $\Phi$ can be written as a current $\phi$, whereas the cyclicity property $\lambda \Phi=\Phi$ imposes that this current is closed $\partial \phi=0$. Actually, it is exactly the same thing in the classical case of commutative calculus.

Notice also that we did not prove that all cyclic cocycles (or cyclic cohomology classes) can be expressed in terms of non-zero closed currents; indeed it is possible to exhibit a non-trivial cyclic cocycle which is trivial as a Hochschild cocycle (i.e. is a Hochschild coboundary). In such a case the corresponding current is zero. This case actually happens only when $p$ is even and the situation is easy to control since there is only one such cyclic cohomology class that we shall call $\tau$.

\subsection{The hierarchy of cocycles $\tau$}

Let $\theta=\left\{\theta^{\mu}\right\}_{\mu \in\{1, \ldots, N\}}$ be a chart in $\operatorname{Gr}(N)$ and $\left\{\theta^{I}\right\}_{I \in\left\{0, \ldots, 2^{N}\right\}}$ denote the corresponding lexicographic basis. We will denote by $\epsilon_{I}$ the dual basis of $\operatorname{Gr}(N)^{*}$, i.e. $\epsilon_{I}\left(\theta^{J}\right)=\delta_{I}^{J}$. Clearly the direct product $(G r(N))^{\times p}$ is spanned by $\epsilon_{I_{1}, I_{2}, \ldots, I_{p}}$, where

$$
\epsilon_{I_{1}, I_{2}, \ldots, I_{p}}\left(\theta^{J_{1}}, \theta^{J_{2}}, \ldots, \theta^{J_{p}}\right)=\delta_{I_{1}}^{J_{1}} \delta_{I_{2}}^{J_{2}} \ldots \delta_{I_{p}}^{J_{p}} .
$$

In section 3 we already called $\tau \doteq \epsilon_{0}$ the fundamental character of $\operatorname{Gr}(N)$, i.e. $\tau$ assigns to each element of the Grassman algebra its "scalar" part. We keep the same notation for

$$
\tau \doteq \epsilon_{0,0, \ldots, 0}
$$

From its definition, we see that, considered as a multilinear form on $\operatorname{Gr}(N), \tau$ vanishes on homogeneous elements unless all elements $a_{i}$ are $\mathbb{C}$-numbers. In this case, $\tau\left(a_{0}, a_{1}, \ldots, a_{p}\right)$ is just equal to the product $a_{0} a_{1} \ldots a_{p}$. From a direct computation, it is clear that $b \tau=0$ if and only if $p$ is even. For example

$$
[b \tau]\left(a_{0}, a_{1}, a_{2}, a_{3}\right)=\left(a_{0} a_{1}\right) a_{2} a_{3}-a_{0}\left(a_{1} a_{2}\right) a_{3}+a_{0} a_{1}\left(a_{2} a_{3}\right)-\left(a_{3} a_{0}\right) a_{1} a_{2}=0
$$

When $p$ is even, $\tau$ is therefore a Hochschild cocycle, moreover, $\tau$ is also clearly cyclic. It is the $\mathrm{b}$ of something (Hochschild cochain) but this Hochschild cochain is not cyclic. Therefore, $\tau$ is a non trivial cyclic cocycle. However, if we try to associate a current to $\tau$, we discover that the corresponding current is strictly zero. What we just computed is nothing else than the cyclic cohomology of complex numbers: it is trivial when $p$ is odd and one- dimensional when $p$ is even. What happens, as illustrated above, is that this hierarchy of cocycles is also part of the cyclic cohomology of Grassmann algebras. For us, this is the uninteresting part and we shall single it out in the sequel. 


\subsection{The dimension of the space of closed currents of degree $p$ on $\operatorname{Gr}(N)$}

For big values of $N$ and $p$, the list of algebraic constraints imposed by the equations $b \Phi=0$ and $\lambda \Phi=\Phi$ becomes rather large $\left(2^{N(p+1)}+2^{N p}\right.$ equations $)$ and a direct attempt of solving these equations would become a formidable task! Fortunately, the fact that we were able to recast these constraints in terms of divergence equations for symmetric tensors will help us. We already gave the general formulae for $\operatorname{dim}^{p}(N)$ in section 3.2 -with $V^{p}(N) \doteq \operatorname{Ker} \partial_{p}$. We now indicate two different proofs of those results.

\section{First method}

The first method rests on an elementary induction proof and on the observation that there exists a vector space isomorphism between $H^{p}$ - that we identify here with the space of $G r(N)$-valued symmetric tensors of order $p$ - and the vector space $V^{p}(N) \oplus V^{p-1}(N)$. Indeed, let $\phi^{\mu_{1} \ldots \mu_{p}}$ a totally symmetric tensor. There are two possibilities: Either $\partial_{\mu_{1}} \phi^{\mu_{1} \ldots \mu_{p}}=0$ or $\partial_{\mu_{1}} \phi^{\mu_{1} \ldots \mu_{p}}=\psi^{\mu_{2} \ldots \mu_{p}} \neq 0$. In the first case, we have $\phi \in V^{p}(N)$. In the second case, $\partial_{\mu_{2}} \psi^{\mu_{2} \ldots \mu_{p}}=-\partial_{\mu_{1}} \partial_{\mu_{2}} \phi^{\mu_{1} \ldots \mu_{p}}=0$ since $\partial_{\mu_{2}} \partial_{\mu_{1}}=-\partial_{\mu_{1}} \partial_{\mu_{2}}$ and $\phi^{\mu_{1} \mu_{2} \ldots \mu_{p}}=\phi^{\mu_{2} \mu_{1} \ldots \mu_{p}}$. Thus $\psi \in V^{p-1}$. Therefore, we have constructed a linear map from $H^{p}$ onto $V^{p-1}$ with kernel $V^{p}$. This establishes the existence of the above vector space isomorphism. Consequently $\operatorname{dim} H^{p}=\operatorname{dim} V^{p}+\operatorname{dim} V^{p-1}$. The induction goes as follows: When $p=0$, the space $H_{\lambda}^{0}$ is just the dual of $\operatorname{Gr}(N)$ - the conditions $b \Phi=0$ and $\lambda \Phi=\Phi$ are trivially satisfied- (actually $\mathcal{C}^{0}=Z^{0}=\mathcal{C}_{\lambda}^{0}=H_{\lambda}^{0}=G r(N)^{*}$.) The dual is, as $\operatorname{Gr}(N)$ itself, of dimension $2^{N}$, and therefore $\operatorname{dim} V^{0}=2^{N}-1$ (since we single out the character $\tau$ ). We already know the dimension of $H^{p}$, namely $2^{N} C_{N+p-1}^{p}$. Therefore

$$
\begin{aligned}
\operatorname{dim} V^{p} & =2^{N}\left\{C_{N+p-1}^{p}-C_{N+p-2}^{p-1}+\ldots+(-1)^{p-1} C_{N}^{1}\right\}-(-1)^{p-1}\left(2^{N}-1\right) \\
& =2^{N}\left\{C_{N+p-1}^{p}-C_{N+p-2}^{p-1}+C_{N+p-3}^{p-2}-\ldots+(-1)^{p} C_{N-1}^{0}\right\}-(-1)^{p} \\
& =2^{N} \gamma_{p}^{N}-(-1)^{p}
\end{aligned}
$$

with $\gamma_{p}^{N} \doteq \sum_{i=1}^{p+1}(-1)^{(i-1)} C_{N+p-i}^{p-(i-1)}$. Symmetric tensors valued in Grassmann algebras are themselves even or odd and we write, as in section 3.2, $V^{p}(N)={ }^{0} V^{p}(N) \oplus{ }^{1} V^{p}(N)$ of dimensions $e_{p} \doteq \operatorname{dim}{ }^{0} V^{p}(N)=2^{N-1} \gamma_{p}^{N}-(-1)^{p}$ and $o_{p} \doteq \operatorname{dim}{ }^{1} V^{p}(N)=2^{N-1} \gamma_{p}^{N}$. A simple exercise of manipulation of formal series shows that

$$
\begin{aligned}
& e(t) \doteq \sum_{p} e_{p} t^{p}=\frac{2^{N-1}-(1-t)^{N}}{(1+t)(1-t)^{N}} \\
& o(t) \doteq \sum_{p} o_{p} t^{p}=\frac{2^{N-1}}{(1+t)(1-t)^{N}}
\end{aligned}
$$

The $\mathbb{Z}_{2}$-graded Poincaré polynomial encodes the dimension of $V^{p}(N)$ and is defined as $P(t) \doteq$ $e(t)+\epsilon o(t)$ where $\epsilon$ is only a formal symbol. This result was already obtained in [5] using Künneth-like formulae, with no reference to currents in Grassmann algebras. Notice that the 
dimension of the space of cyclic cocycles itself differs from $e_{p}$ and $o_{p}$ by a trivial additive constant (the number 1) depending upon the parity of $p$. Indeed $H_{\lambda}^{p}={ }^{0} H_{\lambda}^{p} \oplus{ }^{1} H_{\lambda}^{p}$ with $\operatorname{dim}{ }^{0} H_{\lambda}^{p}=o_{p}$ in all cases but $\operatorname{dim}{ }^{0} H_{\lambda}^{p}=e_{p}$ when $p$ is odd and $\operatorname{dim}{ }^{0} H_{\lambda}^{p}=e_{p}+1$ when $p$ is even.

\section{Second method}

The other method amounts to compute directly the dimension of the space of those symmetric tensors that satisfy the constraint $\partial \phi=0$. This is a rather tedious work but it is interesting because it is quite explicit and can be used to exhibit a basis of cocycles. We start from $\phi^{\mu_{1} \ldots \mu_{p}}\left(\theta^{1} \ldots \theta^{p}\right)$ such that

$$
\sum_{\mu_{1}=1}^{N} \partial_{\mu_{1}} \tilde{\phi}^{\mu_{1} \mu_{2} \ldots \mu_{p}}=0, \forall \mu_{2} \ldots \mu_{p}
$$

We then develop $\phi$ as follows

$$
\phi^{\mu_{1} \ldots \mu_{p}}\left(\theta^{1}, \ldots, \theta^{N}\right)=\sum_{m=0}^{N} \sum_{\nu_{1}<\ldots<\nu_{m}} \phi_{\nu_{1} \ldots \nu_{m}}^{\mu_{1} \ldots \mu_{p}} \partial_{\nu_{m}} \partial_{\nu_{m-1}} \ldots \partial_{\nu_{1}}\left(\theta^{1} \theta^{2} \ldots \theta^{N}\right)
$$

where $\phi_{\nu_{1} \ldots \nu_{m}}^{\mu_{1} \ldots \mu_{p}}$ is now real (or complex) but totally symmetric in $\mu$ 's and totally antisymmetric in $\nu$ 's. The equations to be solved read $\forall m \in 0,1, \ldots(N-1), \forall \mu_{1}<\nu_{1}<\nu_{2}<\ldots<$ $\nu_{m}, \forall \mu_{2}, \mu_{3}, \ldots, \mu_{p}$,

$$
\phi_{\nu_{1} \ldots \nu_{m}}^{\mu_{1} \ldots \mu_{p}}-\phi_{\nu_{2} \ldots \nu_{m} \mu_{1}}^{\nu_{1} \mu_{2} \ldots \mu_{p}}+\phi_{\nu_{3} \ldots \nu_{m} \mu_{1} \nu_{1}}^{\nu_{2} \mu_{2} \ldots \mu_{p}}-\ldots+(-1)^{m} \phi_{\mu_{1} \nu_{1} \ldots \nu_{m-1}}^{\nu_{m} \mu_{2} \ldots \mu_{p}}=0
$$

Notice that for a fix $m$ there are $C_{N}^{m+1} C_{N+p-2}^{p-1}$ equations but they are not independent. A rather laborious analysis shows that, for each value of $0 \leq m \leq N-1$ there are $\sum_{j=1}^{p}(-1)^{j+1} C_{N}^{m+j} C_{N+p-j-1}^{p-j}$ independent equations. This number is equal (cf appendix B) to

$$
\sum_{j=1}^{p}(-1)^{j+1} C_{N}^{m+j} C_{N+p-j-1}^{p-j}=C_{p+m-1}^{m} C_{N+p-1}^{p+m}
$$

The dimension of the space of symmetric tensors is already known to be equal to $2^{N} C_{N+p-1}^{p}$. The dimension of $V^{p}(N)$ is therefore equal to

$$
\operatorname{dim} V^{p}(N)=2^{N} C_{N+p-1}^{p}-\sum_{m=0}^{N-1} C_{p+m-1}^{m} C_{N+p-1}^{p+m}
$$

The sum appearing on the right hand side can be written also as (cf appendix B)

$$
(-1)^{p}+2^{N} \sum_{m=0}^{p-1}(-1)^{m} 2^{m} C_{N+p-1}^{p-1-m}
$$

The fact that the quantity $\operatorname{dim} V^{p}(N)$ is equal to the value given previously (first method) relies on a curious identity (proved in appendix B) between binomial coefficients, namely

$$
\sum_{m=0}^{p-1}(-1)^{m} 2^{m} C_{N+p-1}^{p-1-m}=-\sum_{m=0}^{p-1}(-1)^{m+p} C_{N+m-1}^{m}
$$




\subsection{The case $p=1$}

The case $p=1$ is of particular interest. Indeed, those cocycles can be used to construct several inequivalent cyclic cocycles on the super-circle $C^{\infty}\left(S^{1}\right) \otimes G r(N)$ and this, in turns, allows one to construct many inequivalent central extensions of graded loop algebras (i.e. super Kač-Moody algebras) [3]. Even, in this simple case $p=1$, the total number of independent cyclic one-cocycles grows rapidly with $N$. Previous formulae give $\operatorname{dim}{ }^{0} H_{\lambda}^{1}=(N-1) 2^{N-1}+1$ and $\operatorname{dim}{ }^{1} H_{\lambda}^{1}=(N-1) 2^{N-1}$. Altogether $d=\operatorname{dim} H_{\lambda}^{1}=(N-1) 2^{N}+1$. In the cases $N=1,2,3$, for instance, one gets $d=1, d=3+2 \epsilon \approx 5$ and $d=9+8 \epsilon \approx 17$. For example, in the case $\mathrm{N}=2, \mathrm{p}=1$, the most general current of degree 1 reads $\phi=\phi^{1} \partial_{1}+\phi^{2} \partial_{2}$. Taking into account cyclic symmetry does not bring anything new in this simple case. Let us write $\phi^{i}=\phi_{0}^{i}+\phi_{1}^{i} \theta^{1}+\phi_{2}^{i} \theta^{2}+\phi_{12}^{i} \theta^{1} \theta^{2}$. Then $\tilde{\phi}^{i}=\phi_{0}^{i}-\phi_{1}^{i} \theta^{1}-\phi_{2}^{i} \theta^{2}+\phi_{12}^{i} \theta^{1} \theta^{2}$. The condition $\partial \phi=0$ reads

$$
\begin{aligned}
\partial_{1} \tilde{\phi}^{1}+\partial_{2} \tilde{\phi}^{2} & =0 \\
-\phi_{1}^{1}+\phi_{12}^{1} \theta^{2}-\phi_{2}^{2}-\phi_{21}^{2} \theta^{1} & =0
\end{aligned}
$$

Therefore closed currents are such that $\phi_{1}^{1}+\phi_{2}^{2}=0, \phi_{12}^{1}=0$ and $\phi_{12}^{2}=0$. These three algebraic relations are independent, so we have $8-3=5$ independent cocycles (closed currents). The most general one reads

$$
\phi=\left[x+y \theta^{1}+z \theta^{2}\right] \partial_{1}+\left[x^{\prime}+t \theta^{1}-y \theta^{2}\right] \partial_{2}
$$

Let us take, for instance, $\phi \doteq \theta^{1} \partial_{1}-\theta^{2} \partial_{2}$. Let $a, b \in G r(2)$, with $a=a_{0}+a_{1} \theta^{1}+a_{2} \theta^{2}+a_{12} \theta^{1} \theta^{2}$ (and a similar notation for $b$ ). The bilinear form on $\operatorname{Gr}(2)$ corresponding to $\phi$ is $\Phi(a, b)=$ $\langle\phi, a d b\rangle$ but $d b=\left(b_{1}+b_{12} \theta^{2}\right) d \theta^{1}+\left(b_{2}-b_{12} \theta^{1}\right) d \theta^{2}$ so that $\Phi(a, b)=a_{1} b_{2}+a_{2} b_{1}$. Considering cyclic cocycles as bilinear forms on $\operatorname{Gr}(2)$ and using the notation introduced in eq. (3.12), one can write a basis of this space as follows.

$$
\begin{array}{c|c}
\text { even cocycles } & \text { odd cocycles } \\
\epsilon_{1,1} & \epsilon_{1,12}-\epsilon_{12,1} \\
\epsilon_{2,2} & \epsilon_{2,12}-\epsilon_{12,2} \\
\epsilon_{1,2}+\epsilon_{2,1} &
\end{array}
$$

For instance, $\Phi \doteq \epsilon_{1,2}+\epsilon_{2,1}$ evaluated on $a, b \in G r(2)$, gives $\Phi(a, b)=a_{1} b_{2}+a_{2} b_{1}$ as we already know. We refer to the article [3] for more details concerning the case $p=1$. Since the main subject of the present article is the study of the case $p>1$, we wish now to illustrate the previous techniques by studying an example with $p=2, N=2$. 


\subsection{An explicit example: $\mathrm{N}=2, \mathrm{p}=2$}

An arbitrary current of degree 2 on $G r(2)$ reads $\phi=\phi^{11} \partial_{1} \vee \partial_{1}+\phi^{12} \partial_{1} \vee \partial_{2}+\phi^{21} \partial_{2} \vee \partial_{1}+$ $\phi^{22} \partial_{2} \vee \partial_{2}$. We recall this operator is symmetric, i.e. $\phi^{12}=\phi^{21}$.

Taking into account the symmetry, we see that

$$
\begin{aligned}
\phi^{11} & =\phi_{0}^{11}+\phi_{1}^{11} \theta^{1}+\phi_{2}^{11} \theta^{2}+\phi_{12}^{11} \theta^{1} \theta^{2} \\
\phi^{22} & =\phi_{0}^{22}+\phi_{1}^{22} \theta^{1}+\phi_{2}^{22} \theta^{2}+\phi_{12}^{22} \theta^{1} \theta^{2} \\
\phi^{12}=\phi^{21} & =\phi_{0}^{12}+\phi_{1}^{12} \theta^{1}+\phi_{2}^{12} \theta^{2}+\phi_{12}^{12} \theta^{1} \theta^{2}
\end{aligned}
$$

Then,

$$
\begin{aligned}
\tilde{\phi}^{11} & =\phi_{0}^{11}-\phi_{1}^{11} \theta^{1}-\phi_{2}^{11} \theta^{2}+\phi_{12}^{11} \theta^{1} \theta^{2} \\
\tilde{\phi}^{22} & =\phi_{0}^{22}-\phi_{1}^{22} \theta^{1}-\phi_{2}^{22} \theta^{2}+\phi_{12}^{22} \theta^{1} \theta^{2} \\
\tilde{\phi}^{12}=\tilde{\phi}^{21} & =\phi_{0}^{12}-\phi_{1}^{12} \theta^{1}-\phi_{2}^{12} \theta^{2}+\phi_{12}^{12} \theta^{1} \theta^{2}
\end{aligned}
$$

The condition $\partial \phi=0$ read

$$
\begin{aligned}
\partial_{1} \tilde{\phi}^{11}+\partial_{2} \tilde{\phi}^{21} & =-\phi_{1}^{11}+\phi_{12}^{11} \theta^{2}-\phi_{2}^{12}-\phi_{12}^{12} \theta^{1}=0 \\
\partial_{1} \tilde{\phi}^{12}+\partial_{2} \tilde{\phi}^{22} & =-\phi_{1}^{12}+\phi_{12}^{12} \theta^{2}-\phi_{2}^{22}-\phi_{12}^{22} \theta^{1}=0
\end{aligned}
$$

So we get the constraints

$$
\begin{aligned}
& \phi_{1}^{11}=-\phi_{2}^{12}, \phi_{12}^{11}=\phi_{12}^{12}=0 \\
& \phi_{2}^{22}=-\phi_{1}^{12}, \phi_{12}^{22}=\phi_{12}^{12}=0
\end{aligned}
$$

Notice that we get twice the constraint $\phi_{12}^{12}=0$. A general closed current will therefore depend on the 7 independent parameters $\phi_{0}^{11}, \phi_{1}^{11}, \phi_{2}^{11}, \phi_{0}^{22}, \phi_{1}^{22}, \phi_{2}^{22}, \phi_{0}^{21}$. The most general cyclic cocycle of order 2 on $G r(2)$ reads therefore

$$
\begin{gathered}
\Phi\left(a_{0}, a_{1}, a_{2}\right)=(-1)^{\sigma_{a_{1}}} \quad \int \phi^{i j} a_{0} \partial_{i} a_{1} \partial_{j} a_{2}+k \tau\left(a_{0}, a_{1}, a_{2}\right) \\
=(-1)^{\sigma_{a_{1}}} \quad\left\{\int\left(\phi_{0}^{11}+\phi_{1}^{11} \theta^{1}+\phi_{2}^{11} \theta^{2}\right) a_{0} \partial_{1} a_{1} \partial_{1} a_{2}+\right. \\
\quad \int\left(\phi_{0}^{22}+\phi_{1}^{22} \theta^{1}+\phi_{2}^{22} \theta^{2}\right) a_{0} \partial_{2} a_{1} \partial_{2} a_{2}+ \\
\left.\quad \int\left(\phi_{0}^{12}-\phi_{2}^{22} \theta^{1}-\phi_{1}^{11} \theta^{2}\right) a_{0}\left(\partial_{1} a_{1} \partial_{2} a_{2}+\partial_{2} a_{1} \partial_{1} a_{2}\right)\right\} \\
+k \tau\left(a_{0}, a_{1}, a_{2}\right)
\end{gathered}
$$

where $\tau$ is the canonical cocycle described in section 3.4 and $k$ is an arbitrary constant. All these cocycles are non-trivial, (as cyclic cocycles, i.e. are not cyclic coboundaries since - with the exception of $\tau$ - they can be written as currents) and are inequivalent. By evaluating those Berezin integrals on the elements of a basis $(\theta)^{I}=1, \theta^{1}, \theta^{2}, \theta^{12}$ of $\operatorname{Gr}(2)$ we can rephrase the above results by saying that $H_{\lambda}^{2}=\mathbb{C}^{4} \oplus \mathbb{C}^{4}$ is generated by four even generators and four odd generators that, when expressed as (independent) multilinear forms, with a notation introduced in (3.12), read 


$$
\begin{array}{c|c}
\text { even cocycles } & \text { odd cocycles } \\
\tau=\epsilon_{0,0,0} & \epsilon_{1,1,1} \\
\epsilon_{1,1,12}+\epsilon_{12,1,1}-\epsilon_{1,12,1} & \epsilon_{2,2,2} \\
\epsilon_{2,2,12}+\epsilon_{12,2,2}-\epsilon_{2,12,2} & \epsilon_{1,1,2}+\epsilon_{1,2,1}+\epsilon_{2,1,1} \\
\epsilon_{1,2,12}+\epsilon_{12,1,2}+\epsilon_{2,1,12}+\epsilon_{12,2,1}-\epsilon_{1,12,2}-\epsilon_{2,12,1} & \epsilon_{2,2,1}+\epsilon_{2,1,2}+\epsilon_{1,2,2}
\end{array}
$$

For instance, the second of the above even cocycles is a tri-linear form over $\operatorname{Gr}(2)$ which, when evaluated on a triple $(a, b, c)$ gives $a_{1} b_{1} c_{12}+a_{12} b_{1} c_{1}-a_{1} b_{12} c_{1}$, with $a=a_{0}+a_{1} \theta^{1}+a_{2} \theta^{2}+a_{12} \theta^{1} \theta^{2}$, and similar decompositions for $b$ and $c$.

The above construction using closed currents gives automatically non-trivial cyclic cocycles. This is an important remark. Indeed, if we had tried to solve directly ([4]) the algebraic equations $b \Phi=0$ and $\lambda \Phi=\lambda$ in this case, we would have found that the space of cocycles is actually $Z_{\lambda}^{2}=\mathbb{C}^{5} \oplus \mathbb{C}^{6}$ and is generated therefore by three more multilinear forms that cannot be written as currents. Then, one would have had to show that these objects generate the vector space of cyclic coboundaries, $B_{\lambda}^{2}=\mathbb{C} \oplus \mathbb{C}^{2}$ so that $H_{\lambda}^{2}=\mathbb{C}^{2} \oplus \mathbb{C}^{4}$ as it should. Explicitly, one finds that coboundaries are generated by

$$
\begin{array}{ll}
\epsilon_{0,0,1}+\epsilon_{0,1,0}+\epsilon_{1,0,0} & =b\left(\epsilon_{0,1}-\epsilon_{1,0}\right)=(2 i \pi)^{-1} S\left(\epsilon_{1}\right) \\
\epsilon_{0,0,2}+\epsilon_{0,2,0}+\epsilon_{2,0,0} & =b\left(\epsilon_{0,2}-\epsilon_{2,0}\right)=(2 i \pi)^{-1} S\left(\epsilon_{2}\right) \\
\epsilon_{0,0,12}+\epsilon_{0,12,0}+\epsilon_{12,0,0} & =b\left(\epsilon_{0,12}-\epsilon_{12,0}\right)=(2 i \pi)^{-1} S\left(\epsilon_{12}\right)
\end{array}
$$

The meaning of the $S$ operator in the above expression is explained in appendix A.

\section{Appendix}

\section{A The action of operators $S$ and $B$ in the Grassmannian case}

Several operators called $b, \lambda, A, B_{0}, B, S$ belong to the zoo of non-commutative differential geometry. The first, the Hochschild coboundary operator $b$ was already known before [1]. The others have been invented by [1] to replace and generalize the usual constructions of usual commutative geometry, when the algebra under study is no longer commutative. The case of Grassmann algebra is rather simple and we have been able to study its differential properties without having to introduce the operators $A, B_{0}, B$ and $S$. However, we feel instructive to see what is the meaning of those operators in the present context. The cyclicity operator $\lambda$ was used in the text. $A \doteq 1+\lambda+\lambda^{2} \ldots+\lambda^{N}$ is the cyclic antisymmetrizer; acting on a cyclic object -in our case a closed current on Grassmann algebra- it does essentially nothing here, besides multiplying by a trivial numerical factor. The operator $B_{0}$ is called the non-symmetrized boundary operator, it maps $\mathcal{C}^{p+2}$ to $\mathcal{C}^{p+1}$ and is defined by

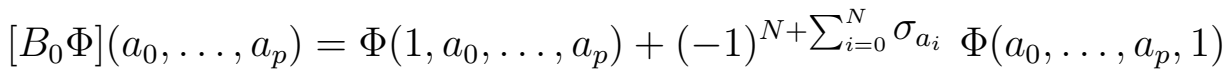


The $\mathrm{B}$ operator is the symmetrized boundary operator; it also maps $\mathcal{C}^{p+2}$ to $\mathcal{C}^{p+1}$ and is defined by $B \doteq A B_{0}$. The main interest of $B$, in general, comes from the fact that $B^{2}=0$ and $B b+B b=0$. Morally, $B$ should be considered - up to a trivial numerical factor- as the non-commutative generalization of the De Rham $d$-operator on forms, or better, since we are dealing with co-chains, as the non-commutative generalization of the De Rham $\partial$-operator acting on currents. This is known to be true in the classical case of commutative geometry, and is clearly valid also in the Grassmaniann context, since, if we consider a closed Hochschild cochain $\Phi$, hence representable as a current $\phi$, we see that $[B \Phi]\left(a_{0}, \ldots, a_{p}\right)$ is proportional to $\left\langle\phi, d a_{0} \vee d a_{1} \vee \ldots \vee d a_{p}\right\rangle$ and is therefore associated with the current $\partial \phi$. In other words, if we had not worked out by hand the definition of the boundary operator $\partial$ we could have used $B$ to define it. This explain also why we did not have to introduce $B$ explicitly before; notice however that action of $B$ is defined on all Hochschild cochains (all multilinear forms on $\operatorname{Gr}(N)$ ) and not only on those that are representable as non-zero currents (the non-trivial Hochschild cocycles). The operator $S$, in our case, brings something new. It is called the Connes' stabilization operator, it maps $C^{p}$ to $C^{p+2}$ and is defined as follows

$$
\begin{aligned}
\frac{1}{2 i \pi} \cdot[S \Phi]\left(a_{0}, a_{1}, \ldots, a_{p}, a_{p+1}, a_{p+2}\right)=\Phi\left(a_{0} a_{1} a_{2}, a_{3}, \ldots, a_{p+2}\right)+ \\
+\sum_{j=2}^{p+1}\left\{\begin{array}{l}
\Phi\left(a_{0}, \ldots, a_{j-1} a_{j} a_{j+1}, \ldots, a_{p+2}\right)+ \\
\left.\quad+\sum_{i=0}^{j-2}(-1)^{j-i+1} \Phi\left(a_{0}, \ldots, a_{i} a_{i+1}, a_{i+2}, \ldots, a_{j} a_{j+1}, a_{j+2}, \ldots, a_{p+2}\right)\right\}
\end{array}\right.
\end{aligned}
$$

For any associative algebra, one shows [1] that $S$ maps cyclic cochains, coboundaries and cocycles of order $p$ to cyclic cochains, coboundaries and cocycles of order $p+2$ and, in particular $H_{\lambda}^{p}$ to $H_{\lambda}^{p+2}$. Also it maps cyclic cochains to Hochschild coboundaries. In particular, if $\Phi \in Z_{\lambda}^{p}$, i.e. $b \Phi=0$ and $\lambda \Phi=\Phi$ then $S \Phi \in Z_{\lambda}^{p+2}$ i.e. $b S \Phi=0$ and $\lambda S \Phi=S \Phi$ but also $S \Phi \in B^{p+2}$ i.e. $\exists \Psi \in \mathcal{C}^{p+1}$ with $S \Phi=b \Psi$. Notice that there is no contradiction because $\Psi$ is not cyclic in general; in other words, if $\Phi$ is a cyclic cocycle $S \Phi$ is always a cyclic cocycle, it may be trivial or not as a cyclic cocycle (as the $b$ of something cyclic) but it is always trivial as a Hochschild cocycle (it is always the $b$ of something). When translated in the particular case of Grassmann algebras, these notions read as follows.

The first observation is that the hierarchy of operators $\tau$ can be obtained from the first $\tau=\epsilon_{0}$ by applying powers of $\mathrm{S}$ :

$$
\epsilon_{2 p}^{\epsilon_{0 \ldots 0}}=\frac{1}{p !} S^{p} \epsilon_{0}
$$

Then, if $\Phi$ is a non trivial cyclic cocycle of order $p$ on $\operatorname{Gr}(N)$, and if we assume that it has no component along $\tau$, it can be considered as a closed current. $S \Phi$ is a priori a cyclic cocycle of order $p+2$ but is is also a Hochshild coboundary and the associated current is zero. However, since $S \Phi$ has no component along $\tau$ and since we have already obtained all the cyclic cocycles of order $p+2$, this implies that $S \Phi$, is not only a Hochschild coboundary but also a cyclic coboundary. In other words, as far as cyclic cohomology is concerned, and with the exception of $\tau$ itself, the hierarchy of cocycles $S^{p} \Phi$ is trivial: we do not get new non-trivial cocycles 
in this way. These remarks explain the (generic) features of the example detailed in section 3.7. To be complete we should mention that the (inductive) limit of $S^{p}\left(H_{\lambda}(G r(N))\right.$ when $p \rightarrow \infty$ is called "periodic cyclic cohomology". The previous analysis shows that, although $H_{\lambda}(G r(N))$ is, by no means, trivial, $H_{\lambda}^{\text {per }}(G r(N))$ reduces to cyclic cohomology of complex numbers.

\section{B Some combinatorial identities}

We start by proving the equality

$$
\sum_{m=0}^{N-1} C_{p+m-1}^{m} C_{N+p-1}^{p+m}=(-)^{p}+2^{N} \sum_{m=0}^{p-1}(-)^{m} 2^{m} C_{N+p-1}^{p-m-1}
$$

Starting with the 1.h.s. of eq. (B.1), one can rewrite it as

$$
\begin{aligned}
\sum_{j=p}^{N+p-1} C_{j-1}^{p-1} C_{N+p-1}^{j} & =\left\{\sum_{j=p}^{N+p-1} C_{N+p-1}^{j} \frac{1}{(p-1) !}\left[(1+t)^{j-1}\right]^{(p-1)}\right\}_{t=0} \\
& =\left\{\left[\frac{1}{(p-1) !} \sum_{j=0}^{N+p-1} C_{N+p-1}^{j}(1+t)^{j-1}\right]^{(p-1)}-\left[\frac{1}{1+t}\right]^{(p-1)}\right\}_{t=0} \\
& =\left\{\left[\frac{1}{(p-1) !} \frac{1}{1+t}(1+(1+t))^{N+p-1}\right]^{(p-1)}\right\}_{t=0}-(-)^{p-1} \\
& =\left\{\frac{1}{(p-1) !} \sum_{m=0}^{p-1} C_{p-1}^{m}(1+t)^{(m)}\left[(2+t)^{N+p-1}\right]^{(p-1-m)}\right\}_{t=0}+(-)^{p} \\
& =(-)^{p}+\left\{\frac{1}{(p-1) !} \sum_{m=0}^{p-1} C_{p-1}^{m} \frac{(-)^{m} m !}{(1+t)^{m+1}} \frac{(N+p-1) !}{(N+m) !}(2+t)^{N+m}\right\}_{t=0}
\end{aligned}
$$

the last equality being just the r.h.s. of eq. (B.1).

Now, we prove the following identity between binomial coefficients:

$$
\sum_{m=0}^{p-1}(-1)^{m} 2^{m} C_{N+p-1}^{p-1-m}=-\sum_{m=0}^{p-1}(-1)^{m+p} C_{N+m-1}^{m}
$$

We first show that

$$
S(N, p)=\sum_{k \leq p} C_{p+N}^{k} x^{k} y^{p-k}
$$

and

$$
S^{\prime}(N, p)=\sum_{k \leq p} C_{k-1+N}^{k} x^{k}(x+y)^{p-k}
$$

are equal. Indeed, they obey the same recursion relation, namely

$$
S(N, p)=(x+y) S(N, p-1)+C_{N+p-1}^{p} x^{p}
$$


This can be proved easily by using the addition theorem for binomial coefficients. Moreover, $S(N, 1)=S^{\prime}(N, 1)$ and $S(N, 0)=S^{\prime}(N, 0)$. Therefore

$$
S(N, p)=S^{\prime}(N, p) \quad \forall N, p
$$

Notice that it can be useful to extend this equality for negative values of $p$ and $N$ by extending the Pascal's triangle upward (i.e. imposing the addition theorem for the $C_{r}^{-s}$ ). By differentiating this equality enough times with respect to $y$, it can also be seen that the above is a remote consequence of the (Pfaff) reflection property (relating arguments $z$ and $\frac{-z}{1-z}$ ) of the Gauss hypergeometric function.

Setting now $x=-1$ and $y=2$ in eq. (B.3), we obtain the equality that we were looking for. It does not seem to be possible to give a simple closed form formula for the above sums, at least with the previous choice for $x$ and $y$. It may be interesting to notice that a nice close formula exists when $x=y=1$ and this is worked out in [10].

We finish with the proof of the equality $(\overline{3.20})$. The demonstration is quite similar to the previous one. We define

$$
\begin{aligned}
S_{1}(p, m) & =\sum_{j=1}^{p}(-)^{j+1} C_{N}^{m+j} C_{N+p-j-1}^{p-j} \\
S_{2}(p, m) & =C_{p+m-1}^{m} C_{N+p-1}^{p+m}
\end{aligned}
$$

(we have drop the dependence in $N$ because it plays no role here). These two binomial coefficient polynomials obey to the same recursion relation, namely

$$
S_{k}(p+1, m)=C_{N}^{m+1} C_{N+p-1}^{p+m}-S_{k}(p, m+1) \quad k=1,2
$$

Moreover, it is easy to compute that $S_{k}(1, m)=C_{N}^{m+1}$, with $k=1,2, \forall m$, so that

$$
S_{1}(p, m)=S_{2}(p, m), \quad \forall m, p .
$$




\section{References}

[1] A. Connes, Non-Commutative Differential Geometry, Publ. Math. IHES 62 (1985).

[2] A. Connes, Noncommutative geometry, IHES/M/93/12 (1993).

[3] R. Coquereaux, L. Frappat, E. Ragoucy, P. Sorba, Extended Kač-moody algebras and their super-derivations algebras, Comm. Math. Phys. 133 (1990) 1-35.

[4] R. Coquereaux, A. Jadczyk, D. Kastler, Differential and Integral geometry of Grassmann algebras, Rev. in Maths. Phys. 3 no 1 (1991) 63-99.

[5] C. Kassel, A Künneth formula for the cyclic cohomology of $\mathbb{Z}_{2}$-graded algebras, Math. Ann. 275 (1986) 683.

[6] J. Dieudonné, Traité d'analyse, Vol 3, Chap. XVII.

[7] F.A. Berezin, Introduction to Superanalysis, MPAM 9, Reidel Pub. (1987), Edited by A.A. Kirillov.

[8] A. Jadczyk, D. Kastler, Graded Lie-Cartan Pairs II Ann. of Phys. 179, n², (1987) 169-199.

[9] D. Kastler, Cyclic cohomology within the differential envelope, Hermann 1988.

[10] R.L. Graham, D.E. Knuth, O. Patashnik, Concrete Mathematics, Addison Wesley 1990. 